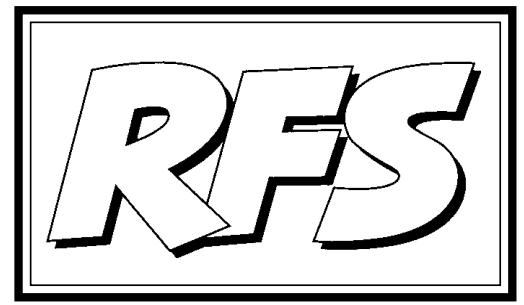

Revista de Fomento Social, 53 (1998), 541-555

\title{
La influencia del estilo directivo en el compromiso organizativo
}

La dirección de personas en las organizaciones ha sufrido un proceso de evolución cuyo futuro parece encontrarse en la gestión de los activos intelectuales -el conocimiento-y emocionales -los valores-. Este último aspecto es el objeto fundamental del presente trabajo. El compromiso individual con la organización constituye, en opinión del autor, un elemento clave que condicionará la gestión de las empresas y organizaciones del próximo siglo. Conocer cuáles son las implicaciones que se derivan de la utilización de un cierto estilo de dirección permitirá, por tanto, diseñar una política eficaz en la gestión del compromiso.

José Antonio ARIZA MONTES(*)

(*) Profesor del departamento de Economía General, Ciencias Jurídicas y Sociología. 


\section{Un nuevo enfoque directivo para un nuevo entorno}

Las organizaciones son entidades sociales creadas deliberadamente para la consecución de unos fines y objetivos de manera racional. Lograr la contribución voluntaria y entusiasta de las personas en la organización es una meta perseguida con empeño por los investigadores del comportamiento humano.

A pesar de esta preocupación casi obsesiva, la dirección ha concentrado tradicionalmente sus estrategias en la inversión en automatización, maquinaria y otras tecnologías que ignoran casi por completo al capital humano. Tales prácticas han demostrado su incapacidad para responder integralmente a los cambios del entorno actual. Al considerar al individuo exclusivamente como un medio a mejorar -y del que se debe extraer el máximo rendimiento- se obstaculiza el desarrollo de sus potencialidades y se imposibilita la aparición de sentimientos de cohesión entre los miembros del grupo y de misión común.

La actuación de los órganos de dirección se ha desarrollado de forma paralela. Así, en el pasado las teorías sobre el management se fundamentaban en el cumplimiento estricto de instrucciones y procedimientos. En este ambiente se transmitían -explícita o implícitamente- valores como la fidelidad, la conformidad, el cumplimiento o la disciplina entre otros. Posteriormente, el interés de los directivos se desplazó hacia la consecución de objetivos que fueran realistas, alcanzables y cuantificables. La racionalización, la motivación o la medición de resultados constituyen algunos de los principales elementos culturales en este tipo de organizaciones.

En la actualidad, la complejidad e incertidumbre del entorno exige un cambio de perspectiva que gire alrededor de los valores. Esta nueva dirección, tal y como advierten García y Dolan (1997: 31), debe estar orientada a rediseñar culturas y facilitar así el gobierno de los cambios estratégicos de la empresa para adaptarse a su entorno y para superar sus tensiones internas. Algunos valores predominantes bajo este nuevo prisma son el desarrollo, la participación, el aprendizaje continuo, la creatividad, la confianza mutua o el compromiso. En este trabajo insistimos en este último valor que consideramos fundamental para la adaptabilidad de la empresa al entorno y creemos, precisamente, que es el estilo de dirección el que debe aglutinar, cohesionar y desarrollar los valores de los individuos. De esta forma, se fomentará una visión o propósito común que generará ilusión, 
esfuerzo y sentido de pertenencia con los objetivos organizativos.

A pesar de los argumentos previos, la política adoptada por la mayor parte de las organizaciones para la resolución del problema de la integración ha sido, durante mucho tiempo, el uso de la disciplina como instrumento de alineación de los objetivos individuales con los organizativos. A lo sumo se han desarrollado sistemas de incentivos económicos vinculados a la consecución de los objetivos. Pese a la innegable utilidad de estos últimos, no creemos que el compromiso se deba fundamentar exclusivamente en la política retributiva.

En esta línea, la dirección debe tener la capacidad de crear y fomentar ideas de futuro, así como de convencer a los demás de que éstas son válidas, justas y legítimas. Lograr que las personas contribuyan a la consecución de los objetivos organizativos requiere el desarrollo de políticas y estrategias que fomenten la actitud favorable de éstas y que libere todas sus energías en pos de la organización. El éxito de este proceso debe concluir en el desarrollo del compromiso personal.

En conclusión, llegar a comprender la influencia del estilo directivo en el fenómeno del compromiso proporcionará una ayuda inestimable para el sistema de gestión y administración de los recursos humanos. En este trabajo sintetizaremos las contribuciones más importantes pero antes haremos una breve referencia sobre qué entendemos por compromiso organizativo.

\section{Estilo directivo y compromiso organizativo}

Las aportaciones teóricas sobre el compromiso organizativo se han desarrollado paralelamente a las investigaciones empíricas que confirman las ventajas que se derivan de fomentar el vínculo individuo-organización. De forma general, Etzioni (1975) considera como compromiso la implicación positiva del individuo. En sentido más estricto se pronuncia Franklin (1975) al referirse a este concepto como el deseo de acatar las normas de la organización y de permanecer en la misma. Porter (1968) lo asocia con el deseo de esforzarse por el bien de la organización, con el anhelo de permanecer en la misma y de aceptar sus principales objetivos y valores. En términos similares se expresan Cook y Wall (1980: 40) al entenderlo como la reacción afectiva de una persona hacia las características de su organización. En concreto se refieren a los sentimientos de apego hacia los objetivos y valores de la organización y a la vinculación con 
la misma por su propio bien más que por una motivación puramente instrumental. Por último, Buchanan (1974) afirma la existencia de tres componentes en el compromiso: identificación o adhesión personal a los objetivos y valores de la organización; implicación entendida como una inmersión psicológica en las actividades del trabajo y, lealtad considerada como un sentimiento de afecto y apego hacia la organización.

En la actitud personal hacia la organización se aprecia una doble dimensión: cuando es de carácter positivo e intensidad fuerte se refiere al compromiso organizativo, mientras que cuando es de naturaleza negativa e igual ímpetu se habla de alienación con respecto a la organización.

La vinculación entre el estilo de dirección y el compromiso organizativo fue enunciada -explícitamente y de forma modelizada- por Etzioni (1975). Este autor considera que existe una relación natural entre las fuentes del poder sobre las que se fundamenta la política directiva-coercitivo, remunerativo o simbólico-y el nivel de implicación -alienativa, calculativa o moral- desarrollado por el individuo (ver figura 1). La implicación moral supondría el reconocimiento de los objetivos y valores de la organización y, por tanto, el compromiso con la misma.

FIGURA 1

Los modelos de Etzioni

\begin{tabular}{|l|c|c|c|}
\hline \multirow{2}{*}{$\begin{array}{l}\text { Formas } \\
\text { de poder }\end{array}$} & \multicolumn{3}{|c|}{ Formas de implicación } \\
\cline { 2 - 3 } & Alienación & Cálculo & Moral \\
\hline Coercitivo & Congruentes & Incongruentes & Incongruentes \\
\cline { 1 - 2 } Remunerativo & Incongruentes & Congruentes & Incongruentes \\
\cline { 1 - 1 } Normativo & Incongruentes & Incongruentes & Congruentes \\
\hline
\end{tabular}

Fuente: Elaboración propia.

Como veremos a continuación, la investigación empírica en este terreno pretende demostrar que en las organizaciones con una dirección más flexible -es decir, donde no se realiza una gestión rígida y estrecha sobre los comportamientos individuales- el compromiso con los objetivos de la organización será ma- 
yor. De esta manera, ciertas prácticas de dirección favorecerán el desarrollo de las conductas comprometidas, mientras que otras obstaculizarán este proceso. Utilizando los pensamientos de Handy (1996: 18) podemos afirmar que en el nuevo lenguaje de la organización uno de los elementos clave es compromiso en lugar de obediencia.

\section{Gestionar el compromiso por medio del estilo de dirección}

Alvin Toffler (1995) se refiere a las diferencias de calidad en las fuentes del poder calificando al coercitivo de malo, al poder remunerativo de medio y señalando como buena la influencia inspirada en valores, ideas y símbolos. De forma similar, Boulding (1993: 71) afirma que en general el poder amenazador [coercitivo] tiende a destruirse a sí mismo a menos que consiga poder integrador [simbólico], es decir, legitimidad, respetabilidad y todo lo demás.

Pese a la presumible virtud que acompaña al estilo directivo apoyado en el poder simbólico, las investigaciones empíricas sobre los efectos originados por la aplicación de las penalizaciones y la distribución de incentivos económicos, también obtienen resultados de signo positivo tal y como veremos en los apartados siguientes.

\subsection{La incapacidad de la disciplina en la gestión del compromiso}

De forma generalizada, los estudios empíricos elaborados sobre los efectos directos que originan las sanciones tienen como finalidad demostrar las consecuencias negativas que se derivan de su aplicación. A pesar de esta línea dominante de análisis, una revisión más profunda descubre otro grupo de investigaciones que sugieren que las sanciones pueden estar igualmente relacionadas de forma positiva con la actuación del subordinado.

Desde esta última perspectiva, algunos estudios han hallado una vinculación directa entre la aplicación de penalizaciones y el aumento de la productividad en la organización, hasta tal punto que Franke y Karl (1978: 636) llegan a afirmar que el uso de este instrumento de control directivo parece haber sido el mayor factor de incremento en los ratios de productividad. Las investigaciones empíricas más interesantes en este sentido las constituyen los estudios de O’Reilly y Weitz (1980), Podsakoff (1982) o Beyer y Trice (1984). Todos ellos contrasta- 
ron que los directivos que empleaban un estilo fundamentado en el uso de las sanciones eran los que obtenían los mejores resultados en sus departamentos.

La relación entre aplicación de sanciones y aumento del compromiso organizativo surge de modo indirecto. Es más, cuando ésta se produce se debe a la intervención de variables intermedias como el nivel de ambigüedad o los sentimientos de equidad. Así, Sims y Szilagyi (1975) demostraron -en una investigación sobre el personal de un hospital- que la aplicación de sanciones servía para reducir las incertidumbres que pudieran existir en relación al desempeño del trabajo. Esta situación se traducía en un aumento de motivación, satisfacción personal y, en última instancia, en un mayor compromiso con la organización. Por otra parte, Schnake y Dumler (1989) observaron que cuando los directivos ignoraban las faltas de los subordinados, se percibían entre los miembros del grupo sentimientos de inequidad: los individuos consideraban que estaban siendo implícitamente penalizados debido a que sus compañeros eran perdonados por comportamientos que deberían ser castigados. La administración justificada de sanciones se convierte de esta forma en un instrumento instigador de los sentimientos de equidad, lo que provoca un mayor compromiso con la organización.

Pese a los intentos por demostrar los efectos positivos que se derivan del empleo de la coerción como mecanismo de gestión directiva, otros argumentos consideran mucho más relevantes los efectos negativos originados por el uso de las penalizaciones. Las evidencias empíricas demuestran que el uso excesivo de la disciplina acarrea efectos contraproducentes que se manifiestan directamente sobre la organización -descensos de productividad, absentismo u otras- o, provocan reacciones individuales -agresividad, insatisfacción...- con repercusión indirecta en la misma.

Uno de los primeros trabajos realizados en este terreno fue el desarrollado por Hamblin (1964). Este autor observó que la aplicación de penalizaciones provocaba un aumento de la agresividad en los subordinados que se traducía en descensos de la productividad y en aumentos de la rotación. Desde otra perspectiva las investigaciones de Hammer y Organ (1978) y O’Reilly y Puffer (1989) comprueban que la aplicación de sanciones provoca insatisfacción, absentismo y una mayor rotación.

Por último, existen numerosas evidencias empíricas que confirman los efectos negativos de las penalizaciones sobre el compromiso organizativo. General- 
mente, estos estudios han demostrado que ante el refuerzo de la coerción se reduce el nivel de compromiso (Etzioni, 1975; Larson y Nelson, 1984; Goffman, 1992; Drummond, 1993; Ariza y Morales, 1997 o Ariza, 1999). Paradójicamente, bajo circunstancias de coerción extrema, las investigaciones de Leighton (1945), Cohen (1953) y el propio Etzioni (1975), coinciden en que la presunta vinculación penalización-compromiso no se manifiesta en la dirección prevista.

\subsection{La eficacia limitada de incentivar a los colaboradores}

La concepción del castigo como instrumento para obtener la conducta deseada implica supuestos sobre la naturaleza humana diferentes de los que fundamentan el efecto motivador de las recompensas. El temor al castigo parece adecuado para prevenir -o impedir-ciertos actos, pero si se persigue un esfuerzo sostenido en el tiempo, los incentivos económicos parecen más efectivos.

La investigación desarrollada en relación al uso de los incentivos de naturaleza económica como mecanismo de dirección parece producir resultados bastante satisfactorios. De esta manera, recompensar el desempeño ocasiona efectos directos e inmediatos sobre el nivel de productividad, la satisfacción, la implicación con el trabajo o, en la línea de este estudio, en el compromiso con la organización.

Así, la investigación desarrollada por Bowey y otros (1982) en cincuenta y dos empresas del Reino Unido entre los años 1977 y 1980 pone de manifiesto el efecto positivo de los sistemas de incentivos sobre la ejecución: el 71\% de las empresas aumentaron su nivel de esfuerzo, el $68 \%$ mejoraron en productividad y, el $85 \%$ consiguió que las ganancias de sus empleados se incrementaran. Estos autores también observaron que en el 55\% de los casos analizados, junto con las mejoras estrictamente cuantitativas se había logrado un aumento en los niveles de calidad. La revisión efectuada por Argyle (1989) -sobre 514 casos de empresas estadounidenses desde el año 1940- descubre de nuevo los efectos positivos de los incentivos económicos sobre los niveles de ejecución (el 39\% de las empresas en las que se implantaron estos sistemas aumentaron su productividad; las ganancias se incrementaron en un 17,5\% y, los costes de producción se redujeron en un 11,5\%). La investigación desarrollada en 1991 por el Instituto de Dirección de Personal (IPM, 1992) ahonda aún más en la relación incentivos-productividad. Los resultados de este estudio ponen de manifiesto que en el 
$74 \%$ de las organizaciones investigadas se utilizaba algún tipo de política de incentivos económicos. La mejora en la actuación organizativa se conseguía en el $62 \%$ de los casos, no obstante, cuando estos mecanismos se complementaban con otras políticas los resultados aumentaban hasta el $95 \%$.

Desde otra perspectiva, la distribución de incentivos ha demostrado provocar un efecto directo sobre la actitud del empleado hacia su trabajo. Por ejemplo, la investigación de Miller (1967) confirmó que los sentimientos de alienación hacia el trabajo disminuyen cuando la organización aplica los sistemas de incentivos de forma habitual. De otra forma, el uso de los incentivos económicos conduce hacia una conducta más implicada con el trabajo.

Otros investigadores se han preocupado por la relación entre incentivos y satisfacción. Así, Podsakoff y otros (1984) observaron que los directivos que administran recompensas y mantienen un adecuado sistema de comunicación interna - de tal forma que los trabajadores perciban que ante una mejora en su rendimiento se producirá un aumento en su gratificación- son los que están rodeados de un personal mucho más satisfecho.

La política de retribución también ha demostrado provocar efectos positivos sobre variables directamente relacionadas con la productividad, como por ejemplo el compromiso organizativo. Desde este punto de vista, Hume (1995) confirma que los sistemas de incentivos facilitan los procesos de cambio organizativo permiten la atracción, reclutamiento y mantenimiento de empleados valiosos y refuerzan el compromiso del empleado con la organización. Osorio y otros (1997: 48) también se pronuncian en este sentido cuando indican que en la actualidad resulta difícil encontrar una compañía que no aspire a lograr el compromiso del personal, la gestión participativa, la mejora de las condiciones de trabajo o el espíritu de equipo. Estos objetivos ofrecen la posibilidad de lograr progresos en la competitividad y el rendimiento de la organización. Sin embargo, en muchas ocasiones estas nuevas metas no están respaldadas por los sistemas retributivos tradicionales, tales como incrementos por méritos, discrecionales, etc. Esta desavenencia puede ser debida a que los anteriores sistemas fueron diseñados para las condiciones de otra época, bajo diferentes criterios económicos, sociales y comerciales y bajo pautas de gestión también diferentes.

La investigación empírica sobre el poder remunerativo y su relación con el compromiso organizativo ha dado lugar a estudios como los desarrollados por Podsakoff y otros (1984), quienes encontraron una vinculación positiva entre 
los incentivos y el compromiso organizativo ( $\mathrm{p}<0,001)$, o Ariza (1999) en el que se encontró una correlación intensa entre ambas variables $(\mathrm{p}<0,001)$ para una muestra de más de mil empleados de diferentes organizaciones.

Pese a la aparente virtud del poder remunerativo, existen indicios prácticos que aconsejan que la gestión del compromiso no se fundamente exclusivamente en los incentivos de naturaleza económica. A veces, en la aplicación de la política retributiva aparecen una serie de inconvenientes que diluyen los efectos positivos atribuidos a este estilo de dirección sobre la aparición del compromiso organizativo.

De esta manera se ha constatado: a) la importancia trascendental que adquiere la equidad en la distribución de los incentivos, al transmitir implícitamente el mensaje de que la contribución del individuo es valorada y reconocida por la organización (Lawler, 1983); b) las preferencias individuales pueden poner en peligro los resultados del sistema (Etzioni, 1975) o; c) la implementación segmentada según los diferentes grupos que integran la organización se debe acomodar a las particularidades del trabajo. Por ejemplo, los sistemas de incentivos en los trabajadores que desarrollan actividades intelectuales se fundamentan en recompensar la actuación individual, mientras que la ocupación manual se retribuye en mayor medida utilizando índices colectivos de ejecución (ACAS, 1988).

Si a estos condicionantes añadimos el efecto a corto plazo de los incentivos debido a que la distribución periódica tiende a transformar la recompensa en un derecho adquirido que pierde toda su capacidad motivadora-comprenderemos por qué es necesario indagar en la existencia de otros mecanismos con una influencia más permanente sobre el compromiso organizativo.

\subsection{La capacidad de la influencia simbólica en la generación de compromiso organizativo}

La influencia obtenida con la utilización de ideas, valores o símbolos se ha relacionado tradicionalmente con el concepto de poder simbólico (Etzioni, 1975) o referente (French y Raven, 1968). Aunque es cierto que este mecanismo constituye un concepto multidimensional de difícil cuantificación, existen motivos sobrados para pensar, tal y como propone Boulding (1993: 129) que, en el fondo, es la más importante de las tres categorías principales del poder. Este 
argumento se fundamenta en la legitimidad que acompaña a esta manifestación del poder, y que es ajena a la influencia ejercida mediante la coerción o el uso de las recompensas por parte de la dirección.

La característica principal del poder simbólico es que se fundamenta en una aceptación voluntaria, legitimada por los individuos. El carácter libre y espontáneo provoca que las relaciones interpersonales en la organización sean de mayor calidad en comparación con los supuestos de control remunerativo y/o coercitivo. El crecimiento de las organizaciones no gubernamentales - donde predomina la integración voluntaria de los miembros y el control basado en el poder simbólico- constituye un claro ejemplo del desplazamiento que se está produciendo en la sociedad actual desde modelos coercitivos hacia diseños organizativos más normativos.

La investigación empírica en este campo confirma que los directivos que cimentan su influencia en el poder de las ideas y los valores están rodeados de personas más satisfechas, menos conflictivas y más disciplinadas y, por supuesto, más comprometidas con la organización.

Desde esta perspectiva, el estudio de Busch (1980) constató que la influencia simbólica estaba positivamente asociada a la satisfacción de los empleados con la dirección, mientras que la aplicación de incentivos económicos no afectaba significativamente a esta variable. Por otra parte, Rahim y Buntzman (1989) comprobaron -con una muestra de 301 personas- que el uso de un estilo de dirección basado en los valores reducía la conflictividad en la organización, al mismo tiempo que aumentaba la sumisión de los empleados. No obstante, la subordinación que logra el poder simbólico permite a estos mantener cierto grado de control sobre su actividad, lo que fomenta la percepción de que más que aceptar la sumisión se está concediendo de forma racional, es decir, se está haciendo un favor a la organización sin ser obligados a ello.

El efecto directo de la dirección fundamentada en valores sobre el compromiso organizativo se aprecia en diferentes investigaciones. Por ejemplo, Drummond (1993) -utilizando una muestra de personas integrantes de diferentes tipos organizativos-demuestra que la influencia de carácter simbólico se traduce en un mayor compromiso con la organización. En nuestro estudio posterior -realizado con una muestra de diferentes organizaciones- se obtuvieron conclusiones similares (Ariza, 1999). Aquellos modelos organizativos que enfatizaban la influencia simbólica eran los que integraban a las personas más comprometidas. 


\section{A modo de conclusión}

En este trabajo se han indicado los principales efectos y la importancia que para las organizaciones actuales supone contar con personas comprometidas. Potenciar los sentimientos de identidad con los objetivos y valores de la organización puede constituir un factor de competitividad y diferenciación determinante para las empresas que aspiren a sobrevivir en un entorno cada vez más cambiante y competitivo.

Pese a su utilidad, las tendencias organizativas de finales de siglo están afectando al compromiso organizacional haciéndolo cada vez menos profundo y más transitorio. Si a ello unimos el ambiente actual -con un trabajo más profesional y un mercado laboral más flexible- entenderemos la reducción en los niveles de cohesión entre los miembros del grupo, y la sustitución de la lealtad del empleado hacia la organización por la lealtad profesional. Aún existen demasiadas organizaciones de nuestro ámbito que prefieren el control a la flexibilidad, utilizando la disciplina como principal mecanismo de control y corrección de las conductas no deseadas. Esta práctica repercute negativamente sobre el nivel de compromiso de los miembros de la organización, lo que dificulta cualquier opción hacia la flexibilización de la estructura.

Aún reconociendo el aumento de contingencias que obstaculizan el desarrollo del compromiso organizativo, nuestra propuesta pretende rehabilitar a este fenómeno como factor de competitividad en la gestión de los recursos humanos. En este sentido hemos constatado que la investigación empírica sobre esta materia parece coincidir en que la mejor forma de dirigir a los individuos hacia los objetivos de la organización es conseguir que estos los interioricen, que los hagan propios, que los compartan, en definitiva, que se sientan comprometidos con la organización. Para conseguir este fin, hemos comprobado que el estilo de dirección más efectivo es aquel fundamentado en las ideas, los valores y el simbolismo. Así se pronuncia Boulding (1993: 60) cuando afirma que existen abundantes pruebas de que el poder integrador [simbólico] es el más importante de los tres, y de que sin él ni el poder militar [coercitivo] ni el poder económico [remunerativo] puede durar mucho tiempo. Aunque en determinadas circunstancias de carácter excepcional el uso de los incentivos económicos -o incluso la distribución equitativa de sanciones-puede provocar el aumento del compromiso con la organización, lo más seguro es conseguir propagar un 
estilo de dirección fundamentado en la influencia simbólica (ver figura 2).

FIGURA 2

Efectos de los diferentes estilos de dirección

\begin{tabular}{|c|c|c|}
\hline & $\begin{array}{c}\text { EFECTOS } \\
\text { POSITIVOS }\end{array}$ & $\begin{array}{c}\text { EFECTOS } \\
\text { NEGATIVOS }\end{array}$ \\
\hline $\begin{array}{c}\text { APLICACIÓN } \\
\text { DESANCIONES }\end{array}$ & $\begin{array}{l}\Delta \text { Productividad } \\
\Delta \text { Equidad } \\
\nabla \text { Ambigüedad } \\
\Delta \text { Compromiso }\end{array}$ & $\begin{array}{l}\nabla \text { Productividad } \\
\triangle \text { Agresividad } \\
\triangle \text { Absentismo } \\
\triangle \text { Rotación } \\
\Delta \text { Insatisfacción } \\
\nabla \text { Compromiso }\end{array}$ \\
\hline $\begin{array}{l}\text { SISTEMADE } \\
\text { INCENTIVOS }\end{array}$ & $\begin{array}{l}\triangle \text { Productividad } \\
\Delta \text { Implicación en el trabajo } \\
\Delta \text { Satisfacción } \\
\Delta \text { Compromiso }\end{array}$ & $\begin{array}{l}\nabla \text { Compromiso } \\
\text { Jactores: } \\
\text { • Problemas de equidad } \\
\text { • Falta de individualización } \\
\text { - Inadecuación con } \\
\text { naturaleza del trabajo }\end{array}$ \\
\hline $\begin{array}{c}\text { DIRECCIÓN } \\
\text { DE VALORES }\end{array}$ & $\begin{array}{l}\triangle \text { Satisfacción } \\
\nabla \text { Conflicto } \\
\triangle \text { Sumisión } \\
\triangle \text { Compromiso }\end{array}$ & \\
\hline
\end{tabular}

Fuente: Elaboración propia.

Las propuestas de este trabajo apuntan hacia una nueva concepción directiva de la organización muy distinta al modelo, más o menos generalizado, fundamentado en la desconfianza y en el control. Por un lado, las personas deberían ser consideradas, tanto en las estructuras como en los procesos, verdadero activo y genuino origen de sus potencialidades. Por otro, el auténtico desafío 
organizativo consiste en crear un ambiente de motivación inducida que potencie el compromiso y libere sus fuerzas motivadoras: la energía, la emoción, el entusiasmo y el esfuerzo en la consecución de los objetivos organizativos.

\section{Bibliografía}

ACAS (1988), Developments in Payment Systems: The 1988ACAS Survey, ACAS, Londres.

Argyle, M. (1989), The Social Psychology of Work, Penguin.

Ariza, J. A. y Morales, A. C. (1997), «Flexibilidad y compromiso: cemento de las estructuras organizativas emergentes», Estudios Financieros, 175, pp. 95-150.

ArizA, J. A. (1999), «Poder y compromiso en las organizaciones», Ed. ETEA (en prensa).

Beyer, J. M. y Trice, H. M. (1984), «A field study on the use and perceived effects of discipline in controlling work performance», Academy of Management Journal, 27 (4), pp. 743-64.

Boulding, K. E. (1993), Las tres caras del poder, Paidós, Barcelona.

Bowey y otros (1982), «Effects of incentive payment systems, United Kingdom (1977-1980)», Department of Employment Research Paper, Reino Unido, 36.

Buchanan, B. (1974), «Building organizational commitment. The socialization of managers in work organizations», Administrative Science Quarterly, 19, pp. 533-46.

Busch, P. (1980), «The sales manager's bases of social power and influence upon the sales force», Journal of Marketing, 44 (4), pp. 91-101.

CoHen, E. A. (1953), Human Endeavour in Concentration Camps, Norton, New York.

Cook, J. D. y WaLl, T. D. (1980), «New work attitude measures of trust, organizational commitment and personal need non-fulfilment», Journal of Occupational Psychology, 53, pp. 39-52.

Drummond, H. (1993), Power and Involvement in Organizations, Avebury, Vermont.

EtZioni, A. (1975), A comparative analysis of complex organizations, The Free Press of Glencoe, New York. 
Franke, R. y KarL, J. (1978), «The Hawthorne experiments revisited: First stadistical interpretation», American Sociological Review, 43, pp. 623-43.

FRANKLIN, J. L. (1975), «Power and commitment-empirical assessment», Human Relations, 28 (8), pp. 737-53.

FRENCH, J. R. y RAVEN, B. (1968), «The bases of social power», en D. Cartwright and A. Zander (Eds.), Group Dynamics, Harper and Row, New York.

García, S. y Dolan, S. L. (1997), La dirección por valores, McGraw-Hill, Madrid.

Goffman, E. (1992), Internados: Ensayos sobre la situación social de los enfermos mentales, Amorrortu editores, Buenos Aires.

Hamblin, R. (1964), «Punitive and non-punitive supervision», Social Problems, 11 , pp. $345-59$.

Hamner, W. C. y Organ, D. W. (1978), Organizational behavior: An applied psychological approach, Business Publications, Dallas.

Handy, C. B. (1996), «El nuevo lenguaje de la labor de organización y sus consecuencias para los líderes», Harvard Deusto Bussines Review, 74, pp. 18-21

Hume, D. A. (1995), Reward Management: Employee Performance, Motivation and Pay, BLACKWELL, Massachusetts.

Institute of Personnel Management (IPM) (1992), Performance Management in the UK: An Analysis of the Issues, Institute of Personnel Management, Londres.

LARSON, J. H. y NelSON, J. (1984), «Women, friendship and adaptation to prison», Journal of Criminal Justice, 12, pp. 601-15.

Lawler, E. E. (1983), Pay and Organisation Development, Addison-Wesley, Massachusetts.

LeIGHTON, A. H. (1945), The governing of men: General principles and recommendations based on experience at a japanese relocation camp, Princeton University Press.

Miller, G. A. (1967), «Professionals in bureaucracy: alienation among industrial scientists and engineers», American Sociological Review, 32, pp. 755-68.

O'REILly, C. A. y Puffer, S. M. (1989), «The impact of rewards and punishments in a social context: a laboratory and field experiment», Journal of Occupational Psychology, 62, pp. 41-53. 
O'ReILly, C. A. y Weitz, B. A. (1980), «Managing marginal employees: The use of warnings and dismissals», Administrative Science Quarterly, 25, pp. 467-84.

Osorio, M., Tovar, P. y Fornieles, A. (1997), «Gain Sharing: Cómo generar y estimular la participación y el compromiso de los empleados mediante la política retributiva», Capital Humano, 98, pp. 45-50.

Podsakoff, P. M. (1982), «Determinants of supervisors use of reward and punishment. A literature review and suggestions for future research», Organizational Behavior and Human Performance, 29, pp. 58-83.

Podsakoff, P. M., Todor, W. D., Grover, B. A. y Guber, O. L. (1984), «Situational moderators of leader reward and punishment behavior-fact or fiction», Organizational Behavior and Human Performance, 34, pp. 21-63.

Porter, L. W. (1968), The Etiology of Organizational Commitment: A Longitudinal Study of Initial Stages of Employee-Organization Relationships, Manuscrito no publicado.

Rahim, M. A. y Buntman, G. F. (1989), «Supervisory power bases, styles of handling conflict with subordinates, and subordinate compliance and satisfaction», The Journal of Psychology, 123 (2), pp. 195-210.

SCHNAKe, M. E. y Dumler, M. P. (1989), «Some unconventional thoughts on the use of punishments in organizations: Reward as punishment and punishment as reward», Journal of Social Behavior and Personality, 4 (1), pp. 97-107.

Sims, H. P. y SzILAGYI, A. D. (1975), «Leader reward behaviour and satisfaction and performance», Organizational Behavior and Human Performance, 14, pp. 426-38.

Toffler, A. (1995), El cambio del poder, P\&J, Barcelona, $3^{\text {a }}$ ed. 